\title{
Ages and metallicities of M31 star clusters ${ }^{\star}$
}

\author{
P. Jablonka ${ }^{1}$, D. Alloin ${ }^{1}$, and E. Bica ${ }^{2}$ \\ ${ }^{1}$ URA 173 CNRS-DAEC, Observatoire de Paris, F-92195 Meudon, Principal Cedex, France \\ ${ }^{2}$ Instituto de Fisica, UFRGS, Caixa Postal 15051, Av. Bento Goncalves, 9500, Porto Alegre, 91500-RS, Brazil
}

Received September 3, accepted November 13, 1991

\begin{abstract}
We present spectrophotometric data for 7 globular clusters and 2 open clusters in M 31. The wide wavelength range available allows one to derive with confidence reddening, metallicity and age. It appears that the foreground and internal reddening are lower than those indicated by previous studies. Evidence of metal content comparable to that in the strongest-lined nuclei of elliptical galaxies is found for the clusters G 158 and G 177, which lie at projected distances less than $1.5 \mathrm{kpc}$ from the galaxy nucleus. The other star clusters show spectral properties similar to those already observed in their Milky Way counterparts of comparable metallicity. The luminous open clusters that we have observed are younger than $30 \mathrm{Myr}$.
\end{abstract}

Key words: galaxies: individual M 31 - clusters: globular, open galaxies: stellar content of - spectroscopy - stars: abundances

\section{Introduction}

The star formation history in a galaxy used to be recovered from its integrated properties through either population or evolutionary spectral syntheses, both involving stellar evolution theory (see for a review, e.g. Alloin et al. 1989). The former approach is limited because of the too many continuity constraints to be taken into account when one populates the composite $\mathbf{H}-\mathbf{R}$ diagram related to the integrated population. The latter approach involves many a priori assumptions, i.e. on the initial mass function or the star formation rate shapes.

A new approach to this problem has been developed by using integrated spectra of clusters as building blocks in population synthesis (Bica \& Alloin 1986a, 1987; hereafter BA86a and BA87, respectively). There are several advantages in it: the number of unknowns is greatly reduced, the age and metallicity of the clusters are known - at least on a relative scale - and other parameters controlling the star formation are inlaid in the clusters automatically. Therefore, a synthesis technique involving a grid of cluster properties as a function of age and metallicity allows one to derive a direct history of the metal enrichment and the relative luminosities of the population components (Bica 1988; hereafter B88). Using the evolutionary synthesis of individual star clusters, which is a relatively tractable problem, it has

Send offprint requests to: D. Alloin

* Based upon data collected at the Canada-France-Hawaii Telescope, partly supported by CNRS, and at the Observatoire de Haute Provence (CNRS). then been possible to recover the mass fractions of different stellar generations in the central regions of nearby galaxies and in global systems when distant cluster galaxies are observed (Bica et al. 1988; Jablonka et al. 1990).

A limitation of this method has been that in the cluster data set there was partial coverage of the age-metallicity plane by the use of star clusters in the Galactic disc, halo and bulge, together with clusters in the Magellanic Clouds. In particular, clusters with spectral features comparable to those in the strongest-lined nuclei of giant elliptical galaxies were not available. The inner bulge clusters we have used, i.e. NGC 6440, NGC 6528 and NGC 6553, have absorption features comparable to those observed in most nuclei of giant galaxies. In order to describe galaxies with extreme absorption features, we have set up an extrapolation procedure of the star cluster integrated properties from $\left[Z / Z_{\odot}\right]=0.1$ to $\left[Z / Z_{\odot}\right]=0.6$ (on the metallicity scale we have adopted).

The present study aims at overcoming the unavoidable uncertainty related to any extrapolation procedure and at extending the cluster data base to higher metallicities, at old, intermediate and young ages if possible. To achieve this goal, we have collected the integrated spectra of clusters in M 31, some of which are already suspected to have an enhanced metal content.

Since Hubble's early list of M 31 possible globular clusters many new candidates have been identified in subsequent searches (see Battistini et al. 1987 for a review). A list of open cluster candidates was provided by Hodge (1979). Integrated $U B V$ and near-infrared photometry is available for many globular clusters (Sharov \& Lyutyi 1983; Bonoli et al. 1987 and references therein). Colours can be used to guess the metallicity and to selecte targets for further detailed spectroscopic analyses; yet this colour information is still lacking for many clusters near the centre of M 31 where the extremely metallic ones are likely to be located.

Various subsamples of the M 31 globular cluster system have been studied spectroscopically in the blue/visual region (e.g. van den Bergh 1969; Burstein et al 1984; Tripicco 1989; Huchra et al. 1991). Burstein et al. and Tripicco have found evidence of systematic differences between M 31 and Galactic globular clusters in some line strengths at comparable metallicities, possibly caused by abundance anomalies or intrmediate age effects. Conversely, Huchra et al. (1991) estimate the metallicity for individual clusters from a set of absorption features, and conclude that the cluster systems are not fundamentally different in $\mathbf{M} 31$ and in our Galaxy.

In the course of the present study, we shall address the problem of metallicity calibrations from integrated properties of globular clusters and their extension to composite stellar popula- 
tions (Aaronson et al. 1978; Zinn \& West 1984; Brodie \& Huchra 1990). Absolute calibrations will remain an open question until the metallicity of individual stars in bulge clusters like NGC 6553 are settled. The basic problem for the moment is still one of ranking. We stress the importance of using a wide spectral range I in order to avoid drawbacks such as dilution effects from hotter stellar components. We also emphasize that it is an oversimplification to apply cluster calibrations through line indices directly to galaxies, which being composite populations, rather require a synthesis analysis.

In Sect. 2 we describe the observational procedure and the data reduction. We present in Sect. 3 the cluster sample, we discuss the foreground reddening and we provide measurements of spectral features and continuum points, performed in a way which is consistent with our previous analyses of cluster integrated spectra. In Sect. 4 we tie the star clusters to our previous grid of cluster properties and estimate their age, and metallicity as well as the reddening from material within M 31 and/or intrinsic to the cluster. Finally, we provide in Sect. 5 a spectral comparison of the strong-lined M 31 star cluster sample with galaxy nuclei and discuss their impact on metallicity rankings and on galaxy spectral syntheses using a base of star clusters. In this section we also discuss what is the origin of differences among the various metallicity scales available. The conclusions of this work are summarized in Sect. 6.

\section{Observational data set and reduction techniques}

Spectrophotometric data were collected with the Herzberg spectrograph at the Cassegrain focus of the $3.6 \mathrm{~m} \mathrm{CFH}$ telescope (Hawaii) from September 25 to 28, 1989. The wavelength coverage was obtained in the overlapping intervals, 3200-5750 A, 5500-7750 ̊ (order sorting filter GG455) and 7500-9750 $\AA$ (order sorting filter RG610). The dispersion was $166 \AA^{\circ} \mathrm{mm}^{-1}$ and the projected scale was 0.4 arcsec $\mathrm{pxl}^{-1}$ on the detector, an RCA CCD preflashed for $600 \mathrm{~ms}$. Aiming at the integrated properties of the M 31 clusters, we had to use rather large entrance slit widths: $1.5,3$ and 4 arcsec, respectively, leading to theoretical spectral resolutions of 8,17 , and $23 \AA$. In practice, most clusters are well concentrated and the effective resolution was better than the nominal value for entrance slit widths of 3 and 4 arcsec. The slit length across the field of view was 1.5 arcmin providing a good estimate of the background, especially for clusters in the bulge of M 31, where it is large and varies rapidly with distance to the centre. In such cases, the slit was set tangentially to the bulge light isophotes so as to minimize underlying light gradients across the cluster. We used an automatic guider all throughout the observing run. A journal of the observations is shown in Table 1. In this table we provide cluster designations in several catalogues: M - Mayall \& Eggen (1953), V - Vetesnik (1962), Bo - Battistini et al. (1987), and G - Sargent et al. (1977). Throughout the paper we will use the latter one. The open cluster designations C 107 and C 130 are from Hodge (1979). In addition to the CFHT observations we also present in this paper a CCD/Carelec spectrum of C 130 taken on August 1987 with the 1.93 m telescope at Haute Provence Observatory (France). The observational and reduction procedures are identical to those employed for the nuclei of M 31, M 32 and NGC 205, obtained in the same run and discussed in Bica et al. (1990a). The reduction of the CFHT observations has been performed in a standard way, with the IRAF/2.7 VMS package. Special attention was given to the background modelling, using 100 pixels on each side of the
Table 1. Journal of observations

\begin{tabular}{lllll}
\hline Object $^{\mathrm{a}}$ & $\begin{array}{l}\text { Slit } \\
(\operatorname{arsec})\end{array}$ & $\begin{array}{l}\text { PA } \\
\left(^{\circ}\right)\end{array}$ & $\begin{array}{l}\text { Exposure }^{\mathrm{b}} \\
n \times(\mathrm{s})\end{array}$ & $\mathrm{AM}^{\mathrm{c}}$ \\
\end{tabular}

\begin{tabular}{|c|c|c|c|c|}
\hline \multicolumn{5}{|c|}{ Nights $25 / 26 / 27$ Sept. $89, \lambda_{0}=4550 \AA$, seeing 0.8 to 1.2} \\
\hline \multirow[t]{2}{*}{ G 1} & 3 & 0 & $1 \times 300$ & 1.07 \\
\hline & 4 & 0 & $2 \times 900$ & 1.07 \\
\hline \multirow[t]{2}{*}{ G 78} & 1.5 & 0 & $3 \times 600$ & 1.08 \\
\hline & 4 & 0 & $3 \times 900$ & 1.12 \\
\hline G 158 & 3 & 0 & $3 \times 1800$ & 1.19 \\
\hline G 170 & 3 & -60 & $3 \times 1800$ & 1.09 \\
\hline G 177 & 3 & -45 & $3 \times 1800$ & 1.39 \\
\hline G 219 & 4 & 0 & $3 \times 1200$ & 1.50 \\
\hline \multirow[t]{2}{*}{ G 222} & 4 & 45 & $1 \times 600$ & 1.15 \\
\hline & & & $2 \times 1200$ & 1.15 \\
\hline C 107 & 3 & -75 & $1 \times 200$ & 2.00 \\
\hline
\end{tabular}

Night 27/28 Sept. 89, $\lambda_{0}=6600 \AA \AA$, $\lesssim 1.0 \operatorname{arcsec}$

$\begin{array}{llrll}\text { G 1 } & 4 & 0 & 1 \times 600 & 1.65 \\ & & & 1 \times 300 & 1.65 \\ \text { G 78 } & 1.5 & 0 & 1 \times 600 & 1.59 \\ & 4 & 0 & 2 \times 600 & 1.49 \\ \text { G } 158 & 3 & 0 & 2 \times 1200 & 1.09 \\ \text { G } 170 & 3 & -60 & 2 \times 1200 & 1.17 \\ \text { G } 177 & 3 & -45 & 2 \times 1200 & 1.08 \\ \text { G 219 } & 4 & 0 & 1 \times 300 & 1.48 \\ & & & 3 \times 600 & 1.48 \\ \text { G } 222 & 4 & 45 & 3 \times 600 & 1.16 \\ \text { C } 107 & 3 & 0 & 1 \times 600 & 1.60\end{array}$

Night $28 / 29$ Sept. $89, \lambda_{0}=8600 \AA$, seeing $1.0-1.2$ arcsec G $1-4 \quad 0 \quad 2 \times 600 \quad 160$ $\begin{array}{lllll}\mathrm{G} 1 & 4 & 0 & 2 \times 600 & 1.60 \\ \mathrm{G} 78 & 1.5 & 0 & 1 \times 1200 & 1.37\end{array}$

$\begin{array}{llll}4 & 0 & 1 \times 1200 & 1.28\end{array}$

$\begin{array}{lllll}\mathrm{G} 158 & 3 & 0 & 1 \times 600 & 1.18\end{array}$

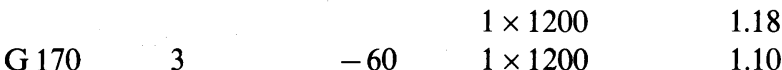

$\begin{array}{llll}\mathrm{G} 177 & 3 & -45 & 1 \times 1200\end{array}$

$\begin{array}{lllll}\mathrm{G} 219 & 4 & 0 & 1 \times 900 & 1.20\end{array}$

$\begin{array}{lllll}\text { G } 222 & 4 & 45 & 1 \times 1200 & 1.08\end{array}$

$\begin{array}{lllll}\mathrm{C} 107 & 3 & 45 & 1 \times 1200 & 1.98\end{array}$

Cluster designations

G1 1

G 78 V $42 \quad$ Bo 23

G 158 V 78 Bo 96

G $170 \quad$ V $56 \quad$ Bo 109

G $177 \quad$ V $67 \quad$ Bo 115

G219 M IV Bo 358

G $222 \quad$ V $87 \quad$ Bo 171

$\mathrm{C} 107$

C 130

${ }^{a}$ The objects numbering is after Sargent et al. (1977) for Globular clusters (G) and after Hodge (1979) for open clusters (C).

${ }^{b}$ The total exposure was split into $n$ successive exposures for optimisation between the signal to noise ratio and the cosmic ray contamination.

${ }^{c}$ Air-mass value at mid exposure.

Two remarks: (1) The seeing was derived as the FWHM of star profiles throughout the night. (2) All along the night the bright hot star HR 234 was observed at various AM for atmospheric absorption monitoring. 
object's spectrum: the appropriate background was subtracted from each spectrum line. In the near-infrared CCD frames, a simple division by the flat field did not permit to remove completely the fringes. In this case the method described in BA 87 was used to remove them. The wavelength calibration was made from Cd-Ne lamp spectra, to a precision better than $2 \AA$. We used usual standard stars for flux calibration and the spectra in each wavelength domain were connected through their overlaps. As our spectral resolution is not suitable for precise velocity determinations, we simply verified their compatibility with that of the M 31 system and rebinned the spectra to rest-frame. The spectra, normalized to the continuum at $5870 \AA$, are described in Sect. 4, where reddening corrections are also discussed.

\section{The star cluster sample and measurements}

\subsection{Sample distribution of the clusters}

We have selected 7 globular clusters in $M 31$ for the present wide spectral range study: G 1, G 78, G 219 and G 222 were chosen so as to have objects in common with previous spectroscopic samples, whereas G 158, G 170 and G 177 were selected because of a suspected extreme metal content from their published colours, or from their central location within M 31. Recently Huchra et al. (1991) have presented a spectroscopic study of 150 M 31 globular clusters in the blue/visual region, where G177 was included: indeed it appeared as one of the most metallic ones.

We show in Fig. 1 the spatial distribution of the clusters studied in this paper, with respect to a sketch of the M 31 disc and bulge, and other objects important for the discussion.

The projected length scale in $\mathrm{kpc}$ is recalled, assuming a distance of $701 \mathrm{kpc}$ for M 31 (Pritchet \& van den Bergh 1984) which corresponds to a distance modulus of $(m-M)_{0}=24.23$, intermediate between the estimates of de Vaucouleurs (1978) and Mould \& Kristian (1986), respectively of 24.07 and 24.4 .

The external contour is the faintest $\mathrm{H} \mathrm{I}$ isophote, according to Roberts \& Whitehurst (1975). It extends up to $30 \mathrm{kpc}$ from the semistellar nucleus along the major axis in the south-west direc-

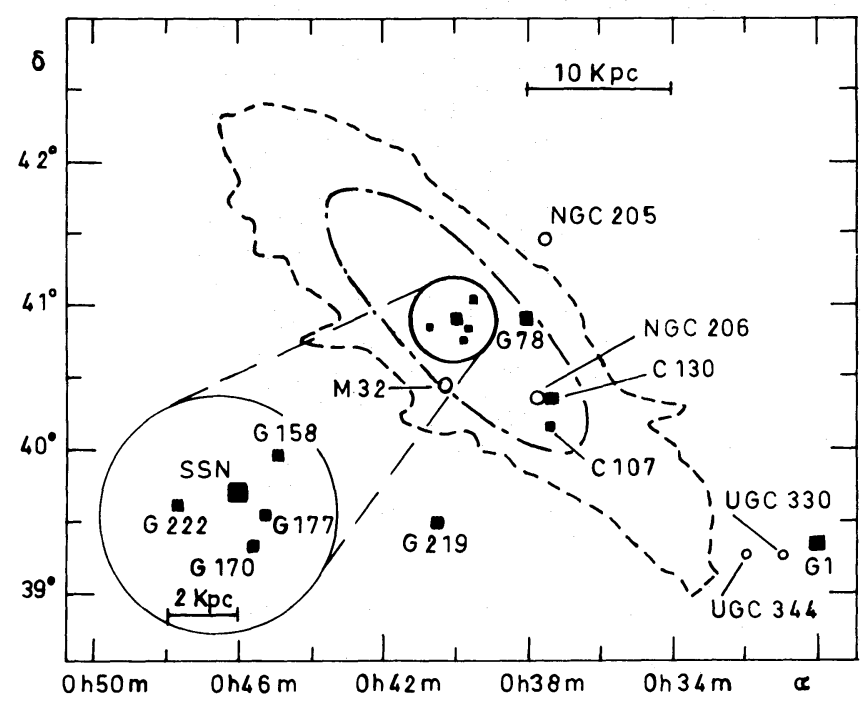

Fig. 1. Spatial distribution of the observed clusters with respect to a sketch of the M 31 disc and bulge. Details in Sect. 3.1 tion. The inner contour is the limit of the M31 disc as normally seen on optical plates.

As a reference, we show the bulge contour at radius $3 \mathrm{kpc}$ with an enlargement on top of the figure. The satellite galaxies M 32 and NGC 205 are shown as well. The dwarf galaxy M 32 is projected on the M 31 disc, while NGC 205 lies along the minor axis, away from the $\mathrm{H} \mathrm{I}$ disc external contour.

The clusters G 158 and G 177 are at projected distances less than $1.5 \mathrm{kpc}$ from the semistellar nucleus of M 31. Owing to their strong metallic features (see Sect. 4), they are most probably associated with the inner bulge, rather than simply projected on it. G 170 and G 222 are as well projected on the bulge and for similar reasons might also belong to it. $\mathrm{G} 78$ is at $\approx 4 \mathrm{kpc}$ from the centre and might be a bulge/halo transition cluster. The halo clusters $G 1$ and $G 219$ are far away, respectively, at projected distances $\approx 30$ and $\approx 16 \mathrm{kpc}$.

The open cluster $\mathrm{C} 130$ is within the borders of the large OB association NGC 206 in the disc of M 31 at a radial distance of $\approx 9 \mathrm{kpc}$ along the major axis, and the open cluster $\mathrm{C} 107$ is located nearby this association.

\subsection{Foreground extinction}

We corrected all cluster spectra for reddening using a normal reddening law $A_{\lambda}=0.49 A_{B}(1 / \lambda-0.35), \lambda$ in $\mu \mathrm{m}$, and the relation $A_{B}=4 E(B-V)$. The foreground Galactic reddening for several galaxies in the field was estimated by Burstein \& Heiles (1984) from Galactic H I column densities. For M 31 and M 32 they found $A_{B}=0,32$ and 0.31 , respectively. For NGC 205, at a slightly lower Galactic latitude, they estimated a much lower value $A_{B}$ $=0.14$. For UGC 344 and UGC 330 located off the $\mathrm{H}$ I disc of M 31 to the south-west, close to the cluster G 1 (Fig. 1), they found $A_{B}=0.16$ and 0.18 , respectively. There is obviously an important increase of the $A_{B}$ estimate for M 32 and M 31 which is probably not of Galactic origin, and might have arisen from a residual contribution of the M 31 disc due to an overlapping of the $\mathrm{H}$ I velocity profiles.

Therefore we adopt for all clusters a Galactic foreground reddening correction $A_{B}=0.16$ or conversely $E(B-V)=0.04$, using NGC 205, UGC 344 and UGC 330 as references, because they are likely not affected by the M $31 \mathrm{H} \mathrm{I}$ component.

Clusters for which the projected distance to the centre of M 31 is small may suffer from additional extinction in the M 31 disc itself or from cluster internal dust extinction (e.g. in disc young clusters). These contributions will be examined in Sect. 4 . The location of the halo clusters G 1 and G 219 suggests that their spectra do not require further reddening corrections.

\subsection{Measurements}

We have measured equivalent widths for a selection of spectral features with continuum tracings as shown in detail in Bica \& Alloin (1988) and window limits as in BA 86a, BA 87. The results are given in Table 2. We provide in Table 3 a set of points measured on the continuum tracings representing the spectral energy distribution of the clusters after the Galactic foreground reddening correction $E(B-V)=0.04$ had been applied.

\section{Ages, metallicity and internal reddening}

The grid of cluster properties consists of the equivalent widths $(W)$ of absorption features and reddening-corrected continuum 
Table 2. Spectral feature equivalent widths

\begin{tabular}{|c|c|c|c|c|c|c|c|c|c|c|c|}
\hline $\begin{array}{l}\text { Window } \\
\text { no. }\end{array}$ & Absorber & $\begin{array}{l}\text { Limits } \\
(\AA)\end{array}$ & G 1 & G 78 & G 158 & G 170 & G 177 & G 219 & G 222 & C 107 & C 130 \\
\hline 2 & $\mathrm{CN}, \mathrm{H} 9$ & $3814-3862$ & 16.4 & 17.4 & 31.1 & 28.5 & 26.5 & 6.4 & 22.3 & 8.3 & 2.8 \\
\hline 3 & $\mathrm{CN}, \mathrm{H} 8$ & $3862-3908$ & 14.2 & 15.6 & 22.1 & 22.3 & 20.2 & 8.8 & 17.5 & 5.1 & 3.8 \\
\hline 4 & $\mathrm{Ca}$ II $\mathrm{K}$ & $3908-3952$ & 15.9 & 17.5 & 21.7 & 19.0 & 21.0 & 7.0 & 20.0 & 3.0 & 2.9 \\
\hline 5 & $\mathrm{Ca}$ II $\mathrm{H}, \mathrm{H} \varepsilon$ & $3952-3988$ & 12.6 & 12.3 & 15.1 & 15.3 & 16.3 & 8.7 & 15.5 & 8.5 & 6.0 \\
\hline 9 & $\mathrm{H} \delta$ & $4082-4124$ & 3.5 & 3.9 & 4.2 & 6.8 & 7.0 & 4.5 & 3.9 & 6.3 & 5.6 \\
\hline 10 & $\mathrm{Fe}_{\mathrm{I}}$ & $4124-4150$ & 2.5 & 2.9 & 5.0 & 6.3 & 6.9 & 0.8 & 4.7 & 0.8 & 0.7 \\
\hline 11 & $\mathrm{CN}$ & $4150-4214$ & 8.0 & 9.4 & 13.8 & 15.9 & 19.0 & 2.2 & 13.5 & 1.2 & 0.0 \\
\hline 12 & $\mathrm{Ca} \mathrm{I}$ & $4214-4244$ & 3.3 & 3.9 & 9.4 & 6.5 & 7.0 & 1.3 & 5.4 & 0.4 & 0.9 \\
\hline 13 & $\mathrm{Fe} I$ & $4244-4284$ & 4.6 & 5.2 & 12.8 & 9.7 & 9.7 & 1.8 & 7.2 & 1.6 & 1.6 \\
\hline 14 & $\mathrm{CH} \mathrm{G}$ & $4284-4318$ & 7.5 & 8.2 & 13.4 & 11.1 & 10.8 & 3.9 & 9.9 & 2.2 & 2.3 \\
\hline 15 & $\mathrm{H} \gamma$ & $4318-4364$ & 5.6 & 5.4 & 7.2 & 7.0 & 5.6 & 5.4 & 6.3 & 7.1 & 7.9 \\
\hline 16 & $\mathrm{Fe}_{\mathrm{I}}$ & $4364-4420$ & 4.7 & 6.1 & 12.8 & 10.7 & 9.4 & 1.6 & 7.9 & 1.9 & 4.7 \\
\hline 27 & $\mathrm{H} \beta$ & $4846-4884$ & 3.3 & 3.6 & 3.5 & 4.2 & 3.9 & 3.5 & 3.2 & 5.6 & 5.9 \\
\hline 31 & $\mathrm{Fe}_{\mathrm{I}}$ & $4998-5064$ & 3.4 & 4.2 & 8.3 & 6.9 & 6.8 & 0.7 & 6.0 & 2.5 & 4.4 \\
\hline 32 & $\mathrm{Fe} \mathrm{I}, \mathrm{C} 2$ & $5064-5130$ & 3.1 & 3.6 & 10.4 & 6.7 & 8.3 & 0.6 & 5.8 & 2.5 & 2.6 \\
\hline 33 & $\mathrm{MgH}, \mathrm{C} 2$ & $5130-5156$ & 1.9 & 2.0 & 5.2 & 3.3 & 3.8 & 0.3 & 3.3 & 1.0 & 1.6 \\
\hline 34 & $\mathrm{Mg}$ I, MgH & $5156-5196$ & 5.0 & 5.5 & 13.2 & 8.8 & 9.8 & 1.0 & 8.1 & 1.8 & 2.2 \\
\hline 35 & $\mathrm{MgH}$ & $5196-5244$ & 2.9 & 3.0 & 6.9 & 4.0 & 5.0 & 0.4 & 5.0 & 1.8 & 3.0 \\
\hline 36 & $\mathrm{Fe}_{\mathrm{I}}$ & $5244-5314$ & 2.8 & 2.5 & 5.7 & 3.7 & 4.3 & 0.5 & 4.8 & 2.1 & 3.3 \\
\hline 48 & $\mathrm{Na} \mathrm{I}$ & $5880-5914$ & 3.2 & 4.5 & 5.6 & 5.7 & 5.8 & 1.7 & 4.8 & 3.3 & 0.6 \\
\hline $54 / 5 / 6 / 7$ & $\mathrm{TiO}$ & $6156-6386$ & 7.3 & 8.9 & 12.8 & 15.0 & 15.9 & 3.1 & 12.8 & 8.2 & 12.4 \\
\hline 60 & $\mathrm{H} \alpha$ & $6540-6580$ & 2.5 & 2.0 & 2.3 & 2.9 & -0.6 & 3.2 & 2.7 & 1.7 & 1.0 \\
\hline $66 / 7 / 8$ & $\mathrm{TiO}$ & $7050-7464$ & 21.1 & 19.0 & 31.4 & 37.3 & 38.7 & 13.7 & 29.7 & 20.1 & - \\
\hline $75 / 76$ & $\mathrm{TiO}, \mathrm{Ti}_{\mathrm{I}}$ & $8234-8476$ & 10.4 & 8.9 & 13.9 & 12.2 & 14.1 & 7.9 & - & - & - \\
\hline 77 & $\mathrm{Ca}$ II, TiO & $8476-8520$ & 2.5 & 2.7 & 5.0 & 4.8 & 6.0 & 2.2 & - & - & - \\
\hline 78 & $\mathrm{Ca}$ II, TiO & $8520-8564$ & 4.5 & 4.4 & 5.8 & 7.0 & 6.1 & 4.1 & - & - & - \\
\hline 80 & $\mathrm{Ca}$ II & $8640-8700$ & 3.8 & 3.9 & 4.9 & 3.8 & 5.6 & 3.0 & - & - & - \\
\hline
\end{tabular}

Notes: (1) Typical errors are less than $10 \%$. (2) Window no. 60 for G 177 is affected by $\mathrm{H} \alpha$ emission and [N II] $6584 \AA$, probably from a local concentration of diffuse ionized gas from the inner disc/bulge in M 31. (3) All values are provided for the entrance slit width of 4 arcsec.

Table 3. Continuum points after correction by the foreground reddening $E(B-V)=0.04$

\begin{tabular}{llllllllll}
\hline$\lambda(\AA)$ & G 1 & G 78 & G 158 & G 170 & G 177 & G 219 & G 222 & C 107 & C 130 \\
\hline 4020 & 0.78 & 0.48 & 0.51 & 0.57 & 0.50 & 1.01 & 0.60 & 2.00 & 2.73 \\
4570 & 0.97 & 0.71 & 0.86 & 0.87 & 0.84 & 1.12 & 0.89 & 1.60 & 2.00 \\
5340 & 1.01 & 0.90 & 0.91 & 0.97 & 0.97 & 1.09 & 1.01 & 1.20 & 1.33 \\
6630 & 0.92 & 1.05 & 1.09 & 0.98 & 1.00 & 0.90 & 0.98 & 0.83 & 0.66 \\
6990 & 0.89 & 1.08 & 1.14 & 0.98 & 0.99 & 0.84 & 0.96 & 0.76 & 0.56 \\
7520 & 0.85 & 1.08 & 1.22 & 1.02 & 1.04 & 0.75 & 0.96 & 0.67 & - \\
8040 & 0.77 & 1.06 & 1.21 & 0.94 & 0.98 & 0.68 & - & - & - \\
8700 & 0.75 & 1.06 & 1.19 & 0.94 & 0.98 & 0.60 & - & - & - \\
\hline
\end{tabular}

Note: Continuum points relative to $F_{5870} \AA$, in $F_{\lambda}$ units. Typical errors are \pm 0.02 .

points mapped as a function of age and metallicity. The results were displayed graphically for the visible range in BA 86a and in a table format, at suitable age and metallicity steps, in Bica \& Alloin (1986b; hereafter BA 86b). For the near-infrared they are gathered in BA87. However, for the present study, we have revisited the $W$ vs. $\left[Z / Z_{\odot}\right]$ relationship, using either a linear regression or a polynomial fit for features with non-linear behavi- our. This was performed separately for globular clusters and clusters younger than $200 \mathrm{Myr}$, which were numerous in the sample, whereas the $W$ vs. $\left[Z / Z_{\odot}\right]$ relations for intermediate ages were interpolated using the observed values of the remaining clusters as a control. A fundamental result was that these relations are split according to the age in the plane $W$ vs. $\left[Z / Z_{\odot}\right]$ for a given metallic feature in the blue, while they progressively tend 
to merge, independently of age, for features at longer wavelengths. This effect is caused by the continuum of hotter main sequence stars in younger clusters which dilutes, in the integrated spectrum, the metallic features towards the blue.

This grid can be applied to new star clusters in order to derive their age, metallicity and reddening. To perform such an analysis, we have established all the necessary $\left[Z / Z_{\odot}\right]$ vs. $W$ equations: the complete set is provided in the Appendix.

\subsection{Discussion on the analysis methods}

Among the different procedures one can follow to make use of the grid in analysing the M 31 globular clusters, we describe the two basic ones:

(a) In the first method, the M 31 globular clusters are assumed to be conventional ones, i.e. to be as old as globular clusters in the Galaxy (ages $>10 \mathrm{Gyr}$ ), and their metallicity is then obtained as an average of $\left[Z / Z_{\odot}\right]$ 's calculated from a selection of metallic features. The $\left[Z / Z_{\odot}\right]$ 's are interpolated in the table grid. This procedure is similar to that usually employed for globular cluster studies (Zinn \& West 1984; Huchra et al. 1991), except that in addition it allows one to test if an M 31 globular cluster is younger than the conventional one: in such a case the metallicity derived from features in the blue/green will be lower than that derived from near-infrared lines, as a consequence of dilution effects. Subequently the correct age can be searched in the grid through comparison with the properties of intermediate age clusters. This method for deriving the age and metallicity is independent of reddening. In turn the reddening can be determined from a comparison of the observed cluster spectral distribution with those of grid points or by means of superposition of template cluster spectra. The selection of metallic features in the blue/green and in the near-infrared ranges, as well as the metallicity derived from each line are given in Table 4 . The average values in each range are also indicated in the table.

(b) The second method is an iterative one, which, by means of minimizations, determines the grid element (age, $\left[Z / Z_{\odot}\right]$ ), whose properties resemble most those of the observed cluster. The grid elements are swept minimizing the differences between observed and grid equivalent widths for a selection of metallic features and Balmer lines from Table 2, as well as for observed (Table 3) and grid continuum points. Reddening corrections are subsequently applied and the procedure is repeated. If no further decrease of the residuals is obtained, the optimal grid element (age, $\left[Z / Z_{\odot}\right]$ ) and reddening value are retained. The method naturally incorporates the relevant observables which discriminate age and metallicity in each grid region: i.e. metallic features in the nearinfrared are basically age independent; Balmer lines are good age discriminators at intermediate and young ages; continuum distribution is an age criterion at intermediate and young ages whereas at fixed old age it becomes a metallicity indicator (BA 86a, BA 87). In practice, we have analysed the metallic features in the visible and near-infrared ranges separately in order to compare their consistency (Table 5).

The latter method does not require an a priori assumption on the age and through successive iterations leads to age, metallicity and reddening estimates simultaneously. However, because of uncertainties inherent to the observations and to the grid construction, the risk cannot be eliminated, as usual in such blind systematic approaches, of ending up with a purely mathematical solution. In the subsequent analysis of globular clusters we use both methods, which should in principle provide similar results.

\subsection{M31 globular cluster analysis}

All clusters, but one, appear to be classical globular clusters as no significant dilution is detected in the blue/green relative to the near-infrared (Tables 4 and 5). The only exception is G 219 for which the average blue/green metallicity is found to be $0.5 \mathrm{dex}$ lower than that derived from the near-infrared region. This could be the signature of an intermediate age or, if it is indeed a classical globular cluster, the features in the blue could be diluted by an anomalously strong blue horizontal branch (HB).

In Figs. 2a and b, G 219 is compared to template star clusters of intermediate and old ages from B 88 . The template $\mathrm{I} 1$ is an average of Galactic disc and LMC clusters in the range 1-2 Gyr,

Table 4. Metallicities derived from the absorption features, assuming the clusters are genuine globulars

\begin{tabular}{|c|c|c|c|c|c|c|c|c|}
\hline Window No. & Feature & G 1 & G 78 & G 158 & G 170 & G 177 & G 219 & G 222 \\
\hline 4 & $\mathrm{Ca}$ II $\mathrm{K}$ & -0.22 & 0.03 & 0.69 & 0.27 & 0.58 & -1.63 & 0.43 \\
\hline 11 & $\mathrm{CN}$ & -0.43 & -0.28 & 0.20 & 0.43 & 0.76 & -1.58 & 0.16 \\
\hline 14 & $\mathrm{CH} \mathrm{G}$ & -0.53 & -0.32 & 1.12 & 0.49 & 0.41 & -1.59 & 0.16 \\
\hline $33+35$ & $\mathrm{MgH}+\mathrm{C} 2$ & -0.63 & -0.56 & 0.73 & -0.06 & 0.19 & -1.77 & 0.11 \\
\hline 34 & $\mathrm{Mg} \mathrm{I}+\mathrm{MgH}$ & -0.60 & -0.45 & 0.99 & 0.24 & 0.41 & -1.88 & 0.12 \\
\hline \multirow[t]{2}{*}{ Mean } & Blue-green & -0.48 & -0.32 & 0.75 & 0.27 & 0.47 & -1.69 & 0.20 \\
\hline & Dispersion & 0.16 & 0.22 & 0.35 & 0.21 & 0.21 & 0.13 & 0.13 \\
\hline $75 / 76$ & $\mathrm{TiO}$ & -0.32 & -0.57 & 0.26 & -0.02 & 0.29 & -0.73 & - \\
\hline 77 & Ca II, TiO & -0.82 & -0.71 & 0.60 & 0.50 & 1.18 & -1.00 & - \\
\hline 78 & $\mathrm{Ca}$ II, TiO & -0.48 & -0.53 & 0.12 & 0.69 & 0.27 & -0.66 & - \\
\hline 80 & $\mathrm{Ca}$ II & -0.70 & -0.64 & -0.05 & -0.71 & 0.35 & -1.19 & - \\
\hline \multirow[t]{2}{*}{ Mean } & Near-IR & -0.58 & -0.61 & 0.23 & 0.12 & 0.52 & -0.90 & - \\
\hline & Dispersion & 0.22 & 0.08 & 0.28 & 0.63 & 0.44 & 0.25 & - \\
\hline
\end{tabular}




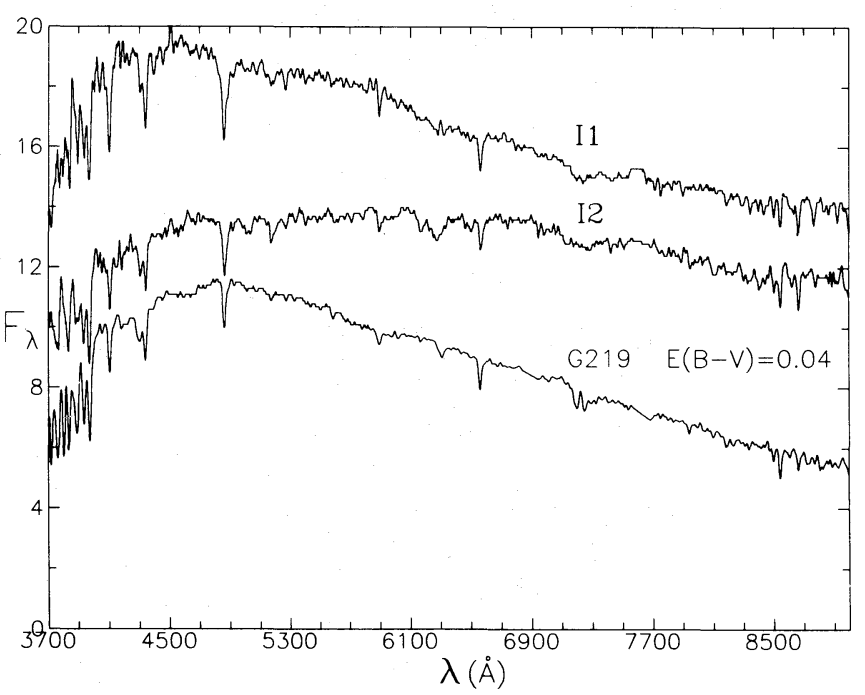

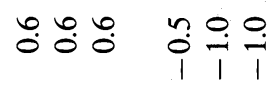

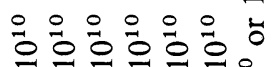

@ б부

吕

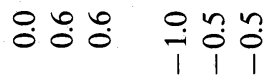

Fig. 2a. Spectrum of $\mathrm{G} 219$ corrected from the foreground reddening $E(B-V)=0.04$ compared to intermediate age templates from the Galactic disc and the LMC. I 1-templ. is an average of clusters whose ages are in the range $1<t(\mathrm{Gyr})<2$, whereas I 2-templ. corresponds to ages in the range $2<t(\mathrm{Gyr})<5$. The spectra, as in the subsequent figures, are normalized to $F_{\lambda}=10$ at $5870 \AA$ continuum level and some shifts are applied for visualisation purposes

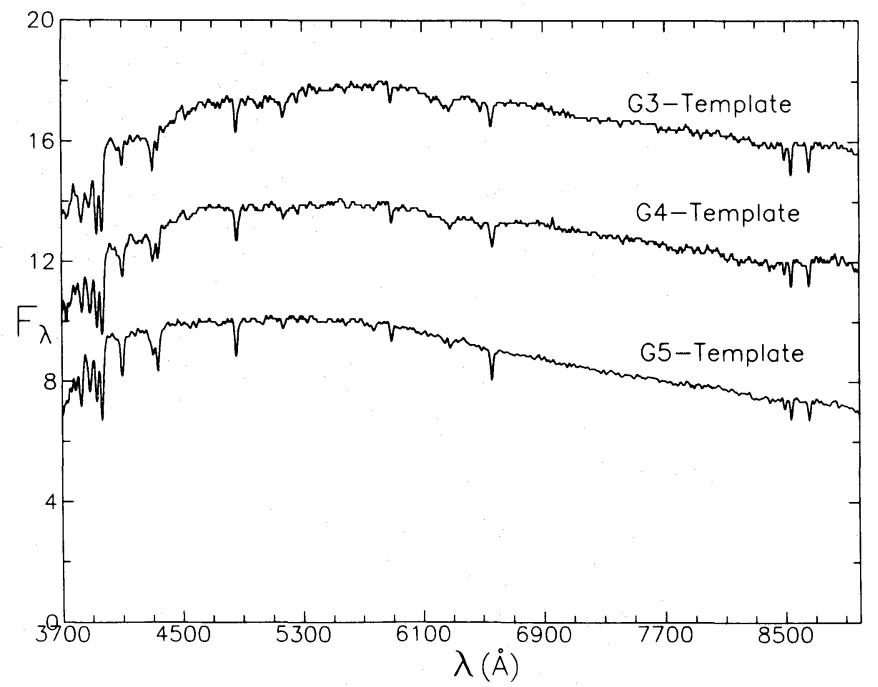

Fig. 2b. Average Galactic Globular cluster spectra whose metallicities are centred at $\left[Z / \mathrm{Z}_{\odot}\right]=-2$ (G 5-templ.), -1.5 (G 4-templ.) and -1 (G 3templ.)

thus with $-1<\left[Z / Z_{\odot}\right]<0$; whereas the template $\mathrm{I} 2$ is a similar average for clusters with ages in the range 2-5 Gyr. The templates G 5, G 4 and G 3 are averages of Galactic Globular clusters with metallicities, respectively, centred at $\left[Z / Z_{\odot}\right]=-2,-1.5$ and -1 . The $\mathrm{G} 219$ continuum resembles that of the I 1-template, but its Balmer lines are more like in the I 2-template. Anyway, the results of the iterative method (Table 5), which suggests that G 219 is of an intermediate age appears to be plausible. The comparison with globular cluster templates (Fig. 2b) indicates that the $\mathrm{Ca}$ II triplet in the near-infrared is like that in the 
$\left[Z / Z_{\odot}\right]=-1$ template, stronger than in the $\left[Z / Z_{\odot}\right]=-2$ one. This shows that G219 (=MIV) is not an extremely metal-poor classical globular cluster like M92 (NGC6341) or M 15 (NGC 7078), contrary to its classification by Burstein et al. (1984) and Huchra et al. (1991): this stresses the risk of relying only on blue/visual spectra. The strongest blue HB in Galactic globular clusters occurs in the $\left[\mathrm{Z} / \mathrm{Z}_{\odot}\right]=-1.5$ template, like the case of M 80 (NGC 6093) (e.g. van Albada 1981). If the dilution in G 219 is caused by a blue $\mathrm{HB}$, it would have to be considerably stronger than in template G 4.

Recently Christian \& Heasley (1991) have produced a VI CCD colour magnitude diagram (CMD) for G 219 , reaching the giant branch, but not the horizontal branch level. They concluded that G 219 is old and extremely metal poor like M 15 . However it is very difficult to observe individual stars in such a compact cluster at the distance of M 31: the scatter is large even for the best observed stars (see their Fig. 3). For a conclusive result on G 219 more observations are necessary. The present analysis suggests that $\mathrm{G} 219$ might be either an intermediate age cluster with $\left[Z / Z_{\odot}\right] \approx-1.0$ or an intermediate metallicity classical globular cluster with an anomalously blue HB.

In order to compare the M 31 metal-rich clusters with those in our Galaxy we use the $\mathrm{G} 1$ and $\mathrm{G} 2$ templates (B88). G1template is an average of NGC 6440, NGC 6528 and NGC 6553, whose metallicities $[\mathrm{Fe} / \mathrm{H}]$ range from -0.29 to 0.12 in Zinn \& West's determination (1984). G 2-template is an average of 47 , Tuc (NGC 104), NGC 5927, NGC 6304, NGC 6316, NGC 6356 (the most metal-rich Galactic globular cluster in Burstein et al. 1984), NGC 6388, NGC 6624, NGC 6637 and NGC 6760, with $-0.74<[\mathrm{Fe} / \mathrm{H}]<-0.30$ on Zinn and West's scale.

Figure 3 shows that the $M 31$ clusters $G 1$ and $G 78$ are very similar to the $\mathrm{G} 2$-template in line strengths. Being already slightly bluer than the G 2-template, G1 requires no internal reddening correction as expected from Sect. 3 . The continuum distribution of $\mathrm{G} 78$ reproduces that of the $\mathrm{G} 2$-template for $E(B$ $-V)_{\mathrm{i}}=0.19$, in agreement with the results of Table 5 . The additional reddening to $\mathrm{G} 78$ suggests that it is behind or embedded

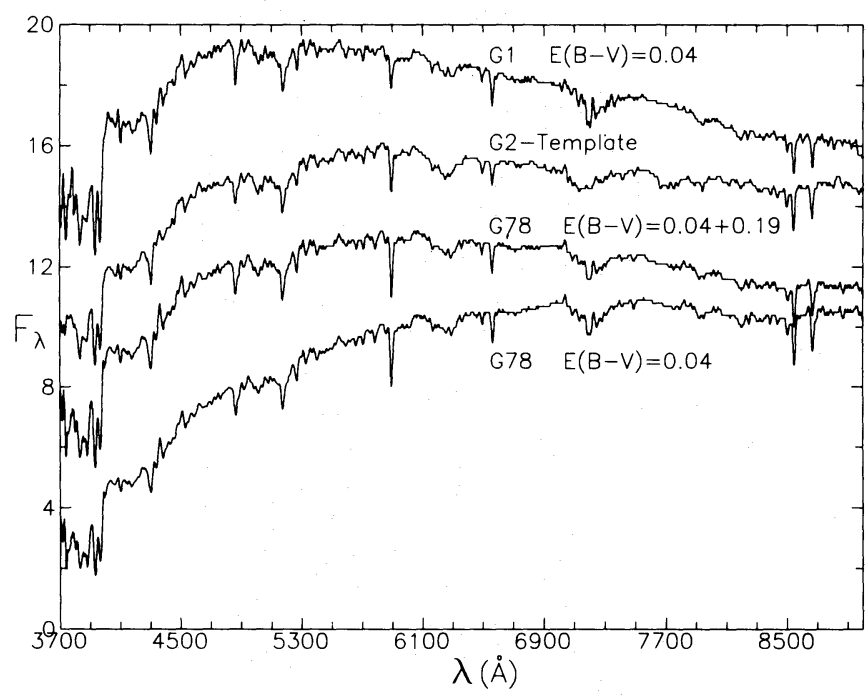

Fig. 3. Spectrum of the M 31 cluster $\mathrm{G} 1$ (corrected from the foreground reddening) compared to the moderately metal-rich $\mathrm{G} 2$-template (e.g. 47 Tuc and NGC 6356 in our Galaxy). The G 78 spectra corrected from the foreground $[E(B-V)=0.04]$ and total $[E(B-V)=0.23]$ reddenings in the disc of M 31. The result that $\mathrm{G} 1$ is similar to the $\mathrm{G} 2$ template (and consequently $47 \mathrm{Tuc}$, one of the less metallic clusters therein) agrees with the VI CMD study of Heasley et al. (1988). They concluded that the giant branch of $\mathrm{G} 1$ was close to that in 47 Tuc. The lower foreground reddening we have adopted of $E(B-V)=0.04$ (Sect. 3) would make even closer the giant branch sequences of the two clusters.

Figure 4 indicates that $\mathrm{G} 222$ and $\mathrm{G} 170$ have properties close to the G1-template (e.g. NGC 6528 and NGC6553 in our Galaxy), and that they do not require internal reddening corrections, in agreement with Table 5. Figure 5a shows that G 177 is more metallic than the G 1-template (Fig. 4) and that its absorption features are comparable to those in the E 1-template, which is an average of the most strong-lined nuclei of elliptical galaxies

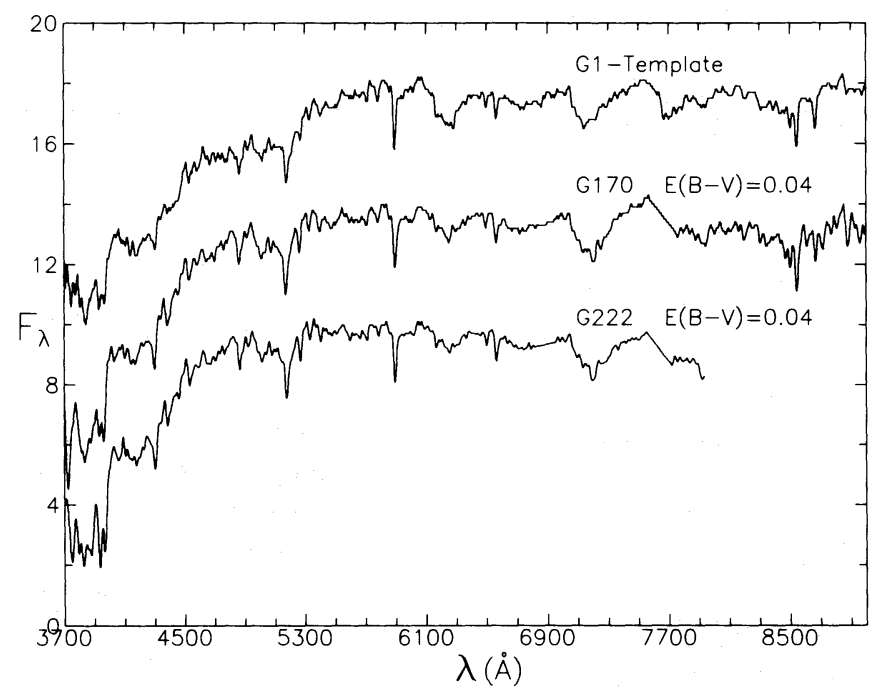

Fig. 4. The spectra of $G 222$ and $G 170$ corrected from the foreground reddening compared to the G1-template (e.g. NGC 6553 in our Galaxy)

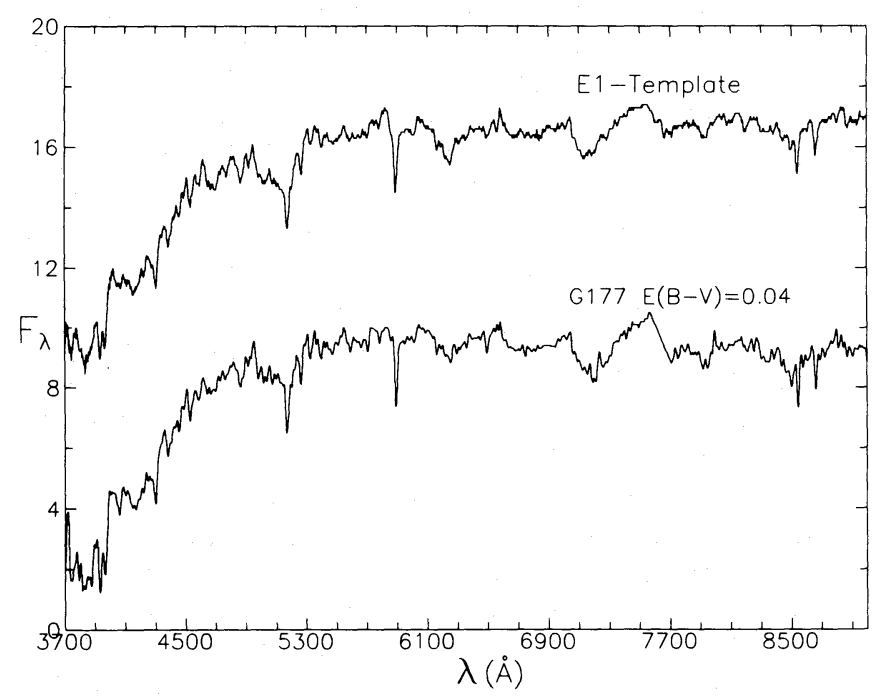

Fig. 5a. Spectrum of G 177 corrected from the foreground reddening and E 1-template, which is an average of the strongest-lined nuclei of elliptical galaxies in Bica (1988) 


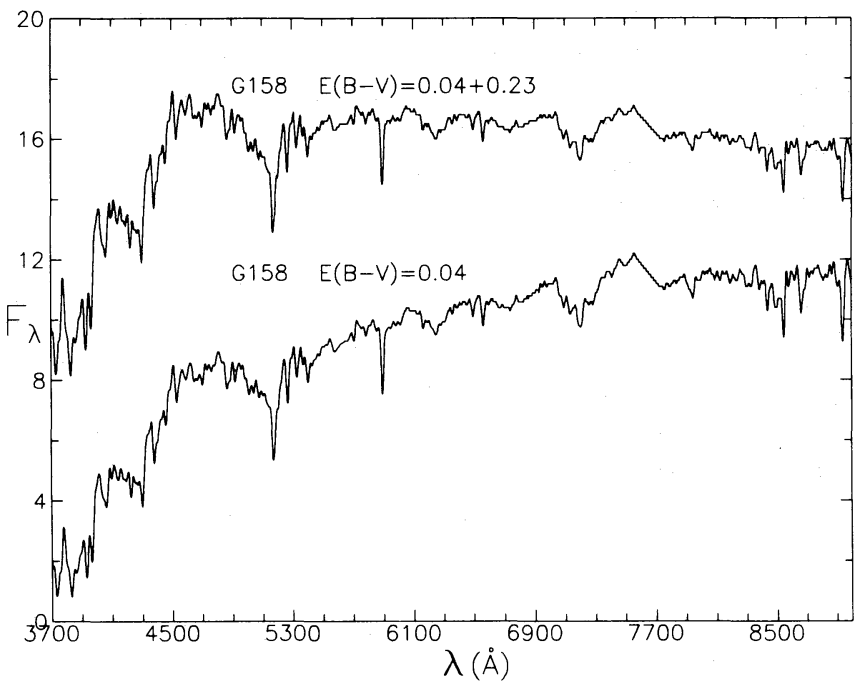

Fig. 5b. G 158 spectra respectively corrected from the foreground $[E(B-V)=0.04]$ and total $[E(B-V)=0.27]$ reddenings

in B 88 and references therein. Its continuum distribution shows that $\mathrm{G} 177$ requires no additional $\mathrm{M} 31$ internal reddening correction.

Figure 5 b shows that G 158 is extreme in metallicity, but it differs in detail from $\mathrm{G} 177$ in some absorption features. It appears to be affected by internal reddening. We display in the figure the result of a reddening correction $E(B-V)_{\mathrm{i}}=0.23$, as obtained in Table 5. This is the correction which makes the red and near-intrared continua of $\mathrm{G} 158$ and $\mathrm{G} 177$ as close as possible. Notice however that, whatever the reddening correction, $\mathrm{G} 177$ has a blue blanketing excess relative to $\mathrm{G} 158$. This fact seems to be related to a difference in their $\mathrm{CNO}$ /iron-peak abundance ratios, which leads one to suspect that $\mathrm{G} 177$ is a genuine inner bulge cluster, whereas G 158 might be an inner disc cluster in M 31 (Bica et al. 1991).

In order to compare quantitatively the M 31 metal rich clusters to those in the Galaxy, according to our $\left[Z / Z_{\odot}\right]$ grid scale, we have applied to the G 2- and G 1-templates the same procedure which generated the metallicities in Table 4 . The average of the blue/green and near-infrared determinations (for G 222 only the former is available) are presented in Table 6 for the Galactic templates and the M 31 clusters. We also show the results for the same objects from Huchra et al. (1991), who tied their M 31 cluster metallicities to Zinn \& West's (1984) $[\mathrm{Fe} / \mathrm{H}]$ scale. It can be concluded that there is a good agreement in the rankings, except that Huchra et al.'s indices, and consequently their derived metallicity, appear to reach saturation for G 177 and $\mathrm{G} 158$. This point as well as the offsets in the metallicity scales are discussed in Sect. 5.

The conclusions that $\mathrm{G} 1$ and $\mathrm{G} 222$ are affected only by foreground reddening, whereas $G 78$ has in addition an M 31 internal reddening, are compatible with those of Burstein et al. (1984) and Frogel et al. (1980), except that our values of the foreground reddening are a factor 2 smaller, and that in the case of $\mathrm{G} 78$ their total reddening is $E(B-V)=0.29$ while ours is 0.23 . The reddening estimates of Crampton et al. (1985) for G 222 , G 177 and G 78 suggest also that only the latter cluster is affected by internal reddening, but their values are too high in all cases.
Table 6. Metallicity rankings. For the Galactic cluster templates $\mathrm{G} 1$ and $\mathrm{G} 2,[\mathrm{Fe} / \mathrm{H}]$ in the last column is the average of the corresponding cluster values in Zinn \& West (1984), whereas for M 31 clusters $[\mathrm{Fe} / \mathrm{H}]$ is from Huchra et al. (1991)

\begin{tabular}{lrl}
\hline Cluster & $\begin{array}{l}{\left[Z / Z_{\odot}\right]} \\
\text { (grid scale) }\end{array}$ & $\begin{array}{l}{[\mathrm{Fe} / \mathrm{H}]} \\
(\mathrm{Zinn} / \text { West scale) }\end{array}$ \\
\hline G 1 & $-0.52 \pm 0.22$ & $-1.08 \pm 0.09$ \\
G 78 & $-0.47 \pm 0.22$ & $-0.92 \pm 0.10$ \\
G 2-templ. & $-0.39 \pm 0.10$ & $-0.54 \pm 0.15$ \\
G 222 & $0.20 \pm 0.20$ & $-0.48 \pm 0.26$ \\
G 170 & $0.20 \pm 0.30$ & $-0.31 \pm 0.78$ \\
G 1-templ. & $0.21 \pm 0.26$ & $-0.14 \pm 0.23$ \\
G 158 & $0.50 \pm 0.37$ & $-0.26 \pm 0.43$ \\
G 177 & $0.52 \pm 0.30$ & $-0.15 \pm 0.38$ \\
\hline
\end{tabular}

Their total reddenings for $\mathrm{G} 158[E(B-V)=0.65]$ and $\mathrm{G} 177$ $[E(B-V)=0.49]$ are exceedingly high relative to ours, which are, respectively, 0.27 and 0.04 . This enormous difference arises from their linear extrapolation to metal-rich clusters of the $S$ parameter vs. intrinsic $(B-V)$ colour relation, which relies only on metal-poor to moderately metal-rich clusters. The spectra in Figs. 4, 5a and 5b show that they have attributed to reddening, colour differences which are instead caused by blanketing effects. However our results still agree in that G 158 is more reddened than G 177 .

\subsection{The open clusters}

The spectra of the M 31 open clusters C 107 and C 130, corrected from the foreground reddening $E(B-V)=0.04$, are displayed in Fig. 6a. They are clearly very blue and consequently are young star clusters. As the near-infrared range was not available, and dilution effects are important at such young ages, we have no direct way of estimating the cluster metallicity. The ages were

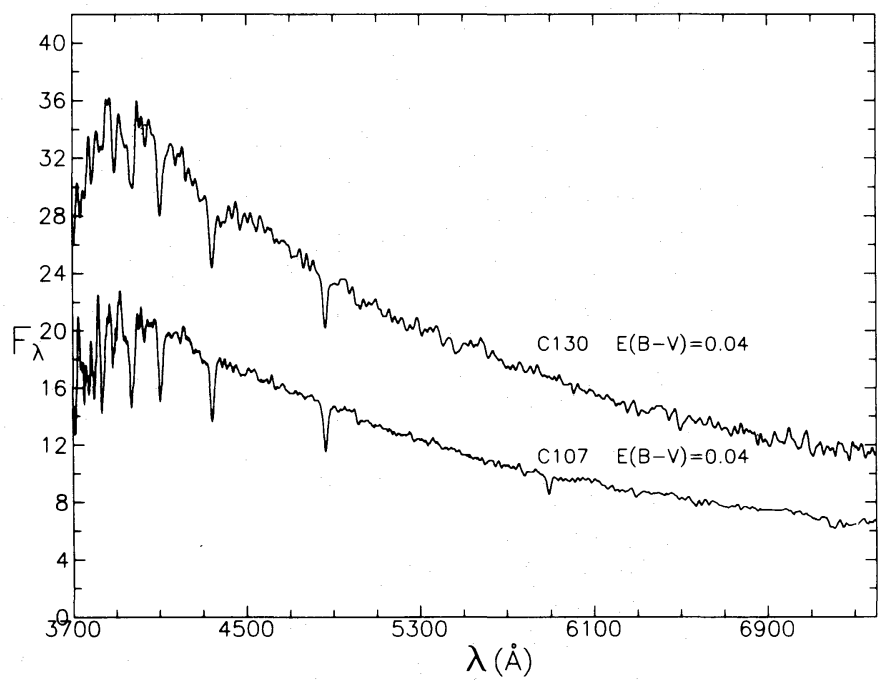

Fig. 6a. Spectra of the M 31 open clusters C 107 and C 130 corrected from the foreground reddening $E(B-V)=0.04$ 


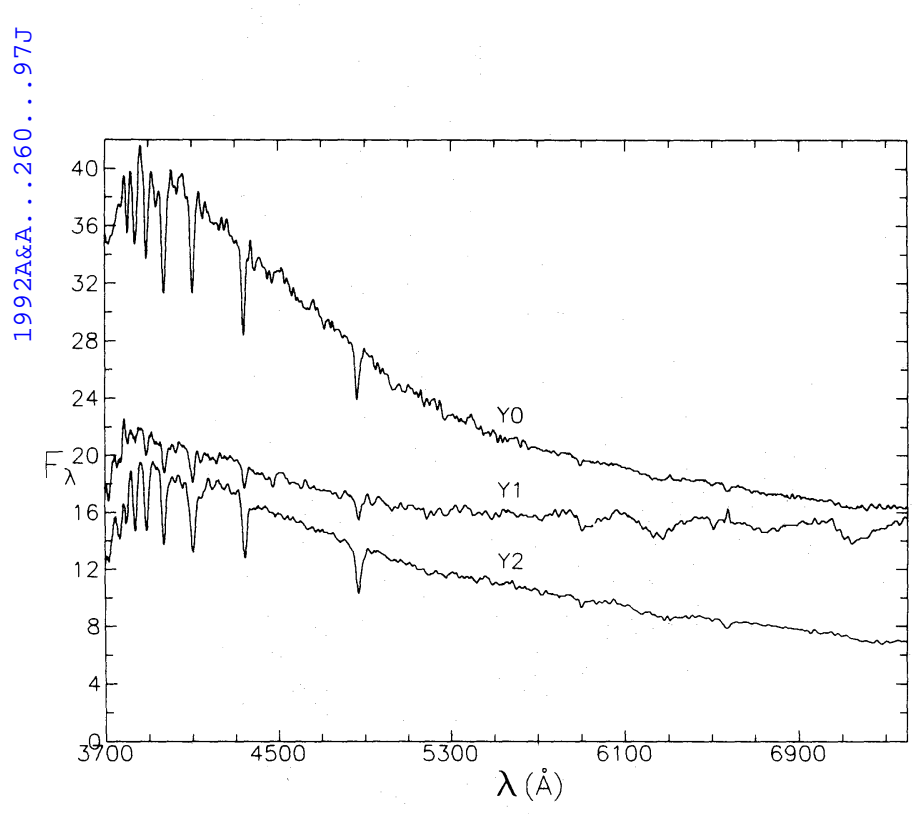

Fig. 6b. LMC averaged clusters corrected from the foreground reddening $E(B-V)=0.06$. Y 0 -templ. is built from clusters of intermediate age (between the age of clusters embedded in $\mathrm{H}$ II regions and the age of clusters in the red supergiant phase); Y 1-templ. shows up the red supergiant phase, while $\mathrm{Y} 2$-templ. is composed of clusters of ages in the range $20<t(\mathrm{Myr})<40$

determined by means of the Balmer lines, which are basically independent of metallicity in young clusters. The observed continuum distribution is used for checking the age and for reddening analyses. Comparisons with base clusters of similar ages show that the dilution of metallic features in $\mathrm{C} 107$ and $\mathrm{C} 130$ are compatible with a solar metallicity.

The equivalent widths of $\mathrm{H} \delta, \mathrm{H} \gamma, \mathrm{H} \beta$ and $\mathrm{H} \alpha$ lines of $\mathrm{C} 107$ and $\mathrm{C} 130$ (Table 2) lead to ages of, respectively, $28 \pm 11$ and $30 \pm 22 \mathrm{Myr}$, interpolating the $W$ vs. age relations with values from the blue domain in the Balmer line grid table of BA 86b.

We show in Fig. 6b the LMC template cluster spectra Y 1 (=NGC 2004) and Y 2 (= NGC 1847+ NGC 2157+ NGC 2214), which represent, respectively, the red supergiant phase at $t \approx 10 \mathrm{Gyr}$ and bluer stages from 20 to $50 \mathrm{Myr}$ (BA 86a, BA 86b; $B$ 88). In addition we present a new average spectrum designated Y 0, which is built up from the clusters NGC 2003 and NGC 1767 in the LMC. Their ages in the range 5-8 Myr are intermediate between those of clusters associated to $\mathbf{H}$ II regions and those in the red supergiant phase, and were studied in the near-infrared in Bica et al. (1990). The visible range spectra of NGC 2003 and NGC 1767 were kindly collected by C. Bonatto and M. Pastoriza in a recent run with the 2D-Frutti detector at the CTIO $1 \mathrm{~m}$ telescope. A comparison of Figs. $6 \mathrm{a}$ and $\mathrm{b}$ indicates that none of the observed M 31 clusters is in the red supergiant phase. The age of $28 \mathrm{Myr}$ derived from the Balmer lines for $\mathrm{C} 107$ is compatible with the characteristics of the Y 2-template. The uncertainty in the age of $\mathrm{C} 130$ does not exclude the possibility of its being as young as the clusters in the Y 0 group, which is suggested by the very blue continuum and the way the spectrum falls off for wavelengths shortward of $4000 \AA$ towards the Balmer jump. Such a young age for $\mathrm{C} 130$ argues for its common origin with the OB association NGC 206, whose global CMD was studied by Odewahn (1987). In particular Odewahn derives for NGC 206 a total reddening of $E(B-V)=0.30$ or 0.23 , respectively, for assumed ages 4 or $10 \mathrm{Myr}$. The fact that $\mathrm{C} 107$ and $\mathrm{C} 130$ are as blue as their possible counterparts in the LMC suggests that no significant internal reddening arises from the disc of M 31. Nevertheless they may suffer some reddening intrinsic to the clusters themselves, just as the young LMC clusters may too. We recall that only a foreground reddening of $E(B-V)=0.06$ was applied to the LMC clusters.

Although strongly diluted, the equivalent width of metallic features in Table 2 for $\mathrm{C} 107$ and $\mathrm{C} 130$ are consistent with those predicted for a solar metallicity at ages $t<30 \mathrm{Myr}$ in the table grid of BA $86 \mathrm{~b}$.

\section{Metal-rich clusters in M 31: impact on stellar populations}

We have compiled in Table 7 equivalent widths for metal-rich stellar populations which, together with metal-rich clusters of M 31 (Table 2), are important to establish rankings. The comparison includes the Galactic Globular cluster templates $\mathrm{G} 2$ and G 1, M 32, the bulge and semi-stellar nucleus of M 31, and nuclei of giant ellipticals averaged in three line-strength groups E 1, E 2, E 3. Figure 7 confirms that the M 31 clusters G 158 and G 177 have absorption feature strengths comparable to those in the E1template or in the semi stellar nucleus of M 31 , which are the strongest-lined populations ever observed. This fact leads to

Table 7. Equivalent widths for other metal-rich populations

\begin{tabular}{|c|c|c|c|c|c|c|c|c|}
\hline & $\begin{array}{l}4 \\
\mathrm{Ca} \text { II K }\end{array}$ & $\begin{array}{l}11 \\
\mathrm{CN}\end{array}$ & $\begin{array}{l}14 \\
\mathrm{CH}-\mathrm{G}\end{array}$ & $\begin{array}{l}33+35 \\
\mathrm{Mg} \mathrm{I}+\mathrm{MgH}\end{array}$ & $\begin{array}{l}34 \\
\mathrm{Mg} \mathrm{I}\end{array}$ & $\begin{array}{l}54-57 \\
\mathrm{TiO}\end{array}$ & $\begin{array}{l}66-68 \\
\mathrm{TiO}\end{array}$ & $\begin{array}{l}78 \\
\mathrm{Ca} I I+\mathrm{TiO}\end{array}$ \\
\hline G 2-template & 14.6 & 7.9 & 7.1 & 5.4 & 5.4 & 12.2 & 19.9 & 4.8 \\
\hline G 1-template & 15.3 & 14.9 & 9.2 & 9.1 & 8.3 & 22.9 & 31.9 & 6.3 \\
\hline M 32 & 17.5 & 8.4 & 8.8 & 7.3 & 7.0 & 19.0 & 27.8 & 6.8 \\
\hline M 31-bulge & 17.6 & 11.5 & 9.2 & 8.9 & 9.3 & 19.2 & 28.7 & 7.2 \\
\hline M 31-SS nucleus & 19.0 & 14.4 & 9.0 & 9.7 & 10.1 & 22.7 & 36.0 & 7.2 \\
\hline E 3-template & 15.3 & 7.3 & 8.6 & 8.1 & 7.7 & 17.4 & 29.1 & 6.6 \\
\hline E 2-template & 17.2 & 10.9 & 9.2 & 9.9 & 9.5 & 19.6 & 28.6 & 6.2 \\
\hline E 1-template & 16.8 & 14.5 & 9.3 & 11.0 & 10.3 & 17.5 & 30.7 & 6.1 \\
\hline
\end{tabular}

Notes: M 31 and M 32 values are from Bica et al. (1990a); some values for the average spectra of elliptical galaxy nuclei are from Bica (1988), the others, as well as those for the average spectra of Galactic Globular clusters, are presented in this paper for the first time. 

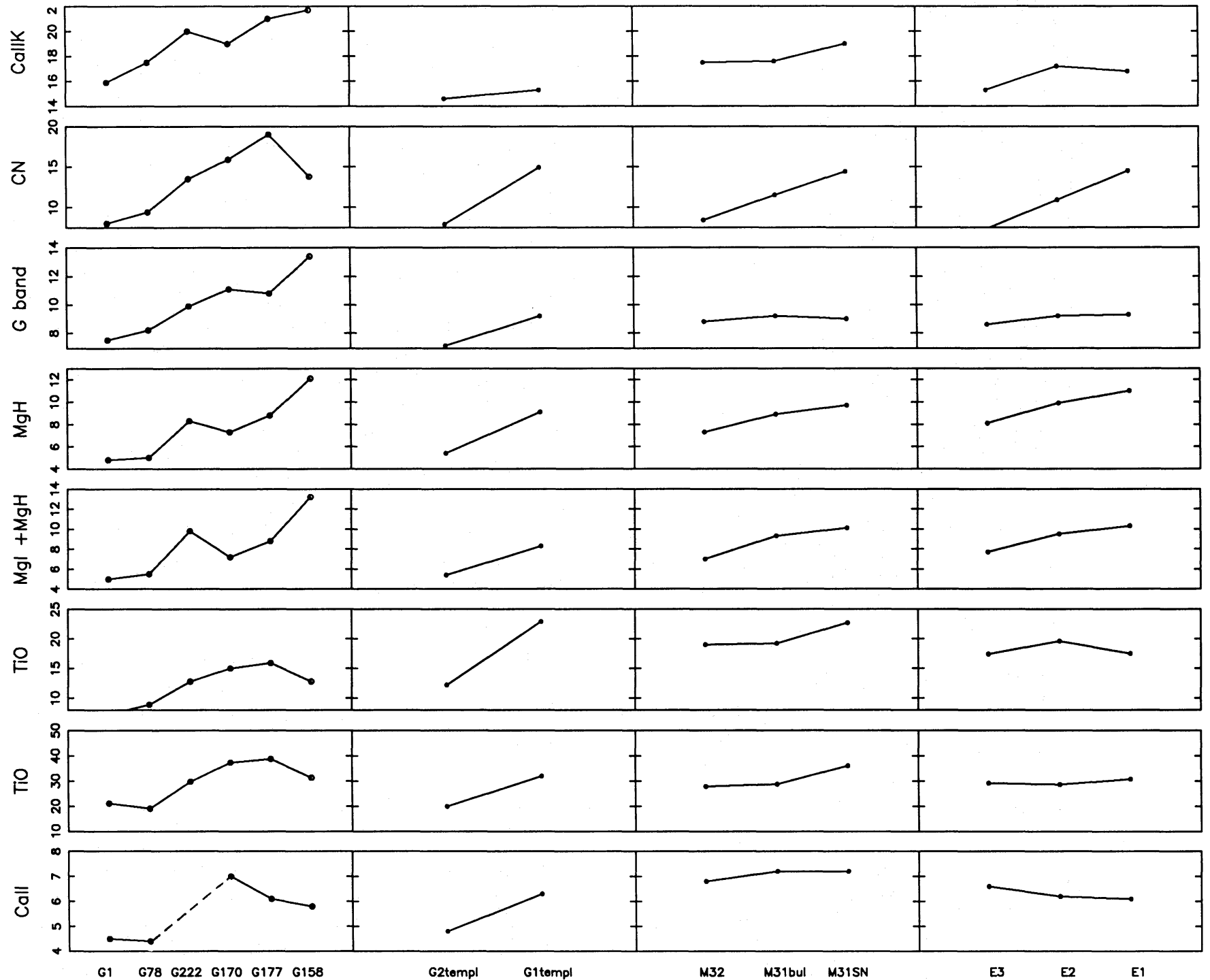

Fig. 7. Comparison of the equivalent widths of metallic features in metal-rich populations

abandon definitely the idea that star cluster metallicities do not overlap with those encountered in giant galaxies, a dogma which lurked the stellar population literature for decades. This wrong notion arose from the fact that the most metal-rich clusters included in earlier samples were like those in the G 2-template or like the M 31 clusters $\mathrm{G} 1$ and $\mathrm{G} 78$.

We also notice from Fig. 7 that G 177 and G 158 differ from each other in a detailed analysis: $\mathrm{CN}$ and $\mathrm{TiO}$ are significantly stronger in $\mathrm{G} \mathrm{177,} \mathrm{while} \mathrm{Mg} \mathrm{I}, \mathrm{MgH}$ and the $\mathrm{G}$ band are weaker. As explained in Bica et al. (1991) this might be related to abundance differences in genuine bulge and inner disc populations.

We conclude that regardless of absolute metallicity calibrations, an extrapolation of the observed spectral properties of star clusters is no longer necessary. However it should be stressed that galaxy nuclei are composite stellar populations and rather than a simple application of observable vs. metallicity relations, they require a thorough synthesis. Indeed, B88 found evidence of dilution effects arising from a range in metallicity and in age in red galaxy nuclei.
The offset in the scales in Table 6 arises primarily from the way the calibrations have been performed. Huchra et al. (1991) tied their values to Zinn's third calibration. In the metal-rich domain, this calibration produces $[\mathrm{Fe} / \mathrm{H}]$ values which are intermediate between his first and second ones (Zinn \& West 1984 and references therein). Our grid scale (BA 86a) is based on cluster metallicities from an average between Zinn \& West's (1984) values and those in Bica \& Pastoriza (1983), which in turn are similar to Zinn's first (higher values) calibration. As Zinn's $Q_{39}$ photometric index measures mainly $\mathrm{Ca}$ II $\mathrm{H}$ and $\mathrm{K}$ while Bica and Pastoriza's photometry measures the $\mathrm{CH}$ G band we have adopted the global metallicity $\left[Z / Z_{\odot}\right]$ notation for the scale. A definite scale for metal-rich globular clusters should include as calibrators bulge clusters like NGC 6553 (Ortolani et al. 1990), with detailed high dispersion analysis of individual stars. It is possible that a calibration based on iron-peak elements differs from a $\mathrm{CNO}$ one, if non-solar $\mathrm{CNO} / \mathrm{Fe}$ ratios are confirmed to exist in the bulge. As all these questions are still open, it is not relevant to discuss absolute differences between the metallicity scales. The important point for the moment is still one of ranking 
and as shown in Table 6, there is a good agreement except at the high metallicity edge.

Let us point out also that our procedure differs from Huchra et al. (1991) and Brodie \& Huchra (1990) in the way the indices are measured. They use high dispersion spectra primarily taken for velocity measurements and necessarily have to use continuum flux side bands very close to the features. Our choice of low dispersion spectra and wide spectral range allowed us to adopt with confidence the continuum tracing method. The sidebands which they have defined for the features $\mathrm{CN}, \mathrm{Ca}$ I, G band and $\mathrm{Mg} \mathrm{I}$ are in zones largely contaminated by absorption when inspected on low dispersion spectra. Their wide sideband indices for $\mathrm{MgH}$ and $\mathrm{H} \Delta$, respectively, include the features $\mathrm{H} \beta$ and $\mathrm{CN}$. Absorptions in sidebands become critical in extreme metallicity objects and may be the reason for the observed saturation in their metallicity relation.

\section{Conclusions}

We have observed 9 clusters in M 31, covering a wide spectral range which allows one to confidently disentangle age, metallicity and reddening effects. The main conclusions of this work are:

(1) Evidence is found that the Galactic foreground reddening to M 31 cannot be higher than $E(B-V)=0.04\left(A_{B}=0.16\right)$. Previous estimates of internal reddening in M 31 for metal-rich globular clusters were overestimated because genuine blanketing effects used to be attributed to reddening.

(2) The luminous open clusters C 107 and C 130 are younger than $30 \mathrm{Myr}$.

(3) G 219 is different from the metal-poor globular clusters M 15 or M 92 in our Galaxy. We find it to be either an intermediate age cluster or an old globular cluster with an anomalous, extremely strong blue horizontal branch.

(4) The cluster G170 is comparable in absorption-line strength to the bulge clusters NGC 6553 and NGC 6528 in our Galaxy. These, in turn, exhibit spectra which are comparable to those observed in most galaxy nuclei.

(5) The inner bulge clusters G 158 and G 177 in M 31 present metallic features as strong as those observed in the strongestlined galaxy nuclei ever observed: i.e. the semi stellar nucleus of M 31 and the nuclei of giant ellipticals. Their observation is of major importance for composite population synthesis, metallicity calibration and for the interpretation of metal-rich stellar systems.

\section{Appendix}

We provide below, for every step in age, the set of equations relating the metallicity to the equivalent width $(W)$, for all features used in the current study.

Lines in the visible range

Globular cluster, age of $1.6510^{10}$

$\begin{array}{ll}\mathrm{Ca} \text { II K } & {\left[Z / Z_{\odot}\right]=-2.75+0.16 W} \\ \mathrm{CN} & {\left[Z / Z_{\odot}\right]=-2.08+0.25 W-5.6310^{-3}(W)^{2}} \\ \mathrm{Ca} \mathrm{I} & {\left[Z / Z_{\odot}\right]=-2.29+0.51 W+1.2710^{-4}(W)^{2}} \\ \mathrm{~Gb} & {\left[Z / Z_{\odot}\right]=-2.76+0.31 W} \\ \mathrm{MgH} & {\left[Z / Z_{\odot}\right]=-2.20+0.38 W-1.1810^{-2}(W)^{2}} \\ \mathrm{Mg} \mathrm{I} \mathrm{MgH} & {\left[Z / Z_{\odot}\right]=-2.29+0.39 W-1.1910^{-2}(W)^{2}} \\ \mathrm{TiO} & {\left[Z / Z_{\odot}\right]=-2.39+0.13 W}\end{array}$

Age of $510^{9}$

$\begin{array}{ll}\text { Ca II K } & {\left[Z / Z_{\odot}\right]=-2.75+0.17 W} \\ \mathrm{CN} & {\left[Z / Z_{\odot}\right]=-2.08+0.26 W-6.2010^{-3}(W)^{2}} \\ \mathrm{Ca}_{\mathrm{I}} & {\left[Z / Z_{\odot}\right]=-2.26+0.52 W+3.3910^{-3}(W)^{2}} \\ \mathrm{~Gb} & {\left[Z / Z_{\odot}\right]=-2.72+0.31 W} \\ \mathrm{MgH} & {\left[Z / Z_{\odot}\right]=-2.19+0.40 W-1.2710^{-2}(W)^{2}} \\ \mathrm{Mg}{ }_{\mathrm{I}} \mathrm{MgH} & {\left[Z / Z_{\odot}\right]=-2.26+0.40 W-1.2210^{-2}(W)^{2}} \\ \mathrm{TiO} & {\left[Z / Z_{\odot}\right]=-2.40+0.13 W}\end{array}$

Age of $10^{9}$

$\begin{array}{ll}\text { Ca II K } & {\left[Z / Z_{\odot}\right]=-2.77+0.20 W} \\ \mathrm{CN} & {\left[Z / Z_{\odot}\right]=-2.08+0.31 W-8.5610^{-3}(W)^{2}} \\ \mathrm{Ca} \mathrm{I} & {\left[Z / Z_{\odot}\right]=-2.26+0.63 W+4.7210^{-3}(W)^{2}} \\ \mathrm{~Gb} & {\left[Z / Z_{\odot}\right]=-2.70+0.37 W} \\ \mathrm{MgH} & {\left[Z / Z_{\odot}\right]=-2.23+0.46 W-1.7210^{-2}(W)^{2}} \\ \mathrm{Mg} \text { I MgH } & {\left[Z / Z_{\odot}\right]=-2.25+0.45 W-1.5010^{-2}(W)^{2}} \\ \text { TiO } & {\left[Z / Z_{\odot}\right]=-2.39+0.13 W}\end{array}$

Age of $510^{\circ}$

\begin{tabular}{ll}
\hline Ca II K & {$\left[Z / Z_{\odot}\right]=-2.74+0.29 W$} \\
CN & {$\left[Z / Z_{\odot}\right]=-2.10+0.49 W-6.0610^{-2}(W)^{2}$} \\
$\mathrm{Ca}_{\mathrm{I}}$ & {$\left[Z / Z_{\odot}\right]=-2.32+1.13 W-2.8410^{-3}(W)^{2}$} \\
$\mathrm{~Gb}$ & {$\left[Z / Z_{\odot}\right]=-2.77+0.64 W$} \\
$\mathrm{MgH}$ & {$\left[Z / Z_{\odot}\right]=-2.24+0.61 W-2.6910^{-2}(W)^{2}$} \\
$\mathrm{Mg}$ I MgH & {$\left[Z / Z_{\odot}\right]=-2.27+0.61 W-1.4410^{-2}(W)^{2}$} \\
TiO & {$\left[Z / Z_{\odot}\right]=-2.37+0.14 W$}
\end{tabular}

Ages of $10^{8}$ and $510^{7}$

$\begin{array}{ll}\text { Ca II K } & {\left[Z / Z_{\odot}\right]=-2.89+0.75 W} \\ \mathrm{CN} & {\left[Z / Z_{\odot}\right]=-2.09+1.24 W-0.12(W)^{2}} \\ \mathrm{Ca} \mathrm{I}_{\mathrm{Gb}} & {\left[Z / Z_{\odot}\right]=-2.26+0.52 W+1.35(W)^{2}} \\ \mathrm{~Gb} & {\left[Z / Z_{\odot}\right]=-2.59+2.00 W} \\ \mathrm{MgH} & {\left[Z / Z_{\odot}\right]=-2.27+0.87 W-1.4610^{-2}(W)^{2}} \\ \mathrm{Mg}_{\mathrm{I}} \mathrm{MgH} & {\left[Z / Z_{\odot}\right]=-2.18+0.86 W-1.2210^{-3}(W)^{2}} \\ \text { TiO } & {\left[Z / Z_{\odot}\right]=-2.36+0.15 W}\end{array}$

Lines in the near-infrared, not age-dependent

TiO Ti I $\left[Z / Z_{\odot}\right]=-2.03+0.16 \mathrm{~W}$

Ca II $8498 \quad\left[Z / Z_{\odot}\right]=-2.25+0.58 W-1.7610^{-3}(W)^{2}$

Ca II $8542\left[Z / Z_{\odot}\right]=-2.53+0.44 W+2.9810^{-3}(W)^{2}$

Ca II $8662\left[Z / Z_{\odot}\right]=-2.91+0.56 W+4.6810^{-3}(W)^{2}$

\section{References}

Alloin D., Arimoto N., Bica E., 1989, in: Beckman J.E., Pagel B.E.J. (eds.) Evolutionary Phenomena in Galaxies. Cambridge University Press, Cambridge, p. 409

Aaronson M., Cohen J., Mould J., Malkan M., 1978, ApJ 223, 824

Battistini P., Bonoli F., Braccessi A., Federici L., Fusi Pecci F., Marano B., Borngen F., 1987, A\&AS 67, 447

Bica E., 1988, A\&A 195, 76

Bica E., Alloin D., 1986a, A\&A 162, 21

Bica E., Alloin D., 1986b, A\&AS 66, 171

Bica E., Alloin D., 1987, A\&A 186, 49

Bica E., Alloin D., 1988, in: Kron R.G., Renzini A. (eds.) Towards Understanding Galaxies at Large Redshifts. Kluwer, Dordrecht, p. 77 
Bica E., Pastoriza M., 1983, ApSC 91, 99

Bica E., Arimoto N., Alloin D., 1988, A\&A 202, 8

Bica E., Alloin D., Schmidt A., 1990a, A\&A 228, 23

Bica E., Alloin D., Santos Jr. J.F.C., 1990b, A\&A 235, 103

Bica E., Jablonka P., Santos Jr. J.F.C., Alloin D., Dottori H., 1992, A\&A 260, 109

Bonoli F., Delpino F., Federici L., Fusi Pecci F., 1987, A\&AS 185,25

Brodie J., Huchra J., 1990, ApJ 362, 503

Burstein D., Heiles C., 1984, ApJS 54, 33

Burstein D., Faber S., Gaskell C., Krumm N., 1984, ApJ 287, 586

Christian C.A., Heasley J.N., AJ 101, 848

Crampton D., Cowley A., Scade D., Chayer P., 1985, ApJ 288, 494

de Vaucouleurs G., 1978, ApJ 223, 730

Frogel J.A., Persson S.E., Cohen J.G., 1980, ApJ 240, 785

Heasley J.N., Christian C.A., Friel E.D., Janes K.A., 1988, AN 96, 1312
Hodge P., 1979, AJ 84, 744

Huchra J., Brodie J., Kent S., 1991, ApJ 370, 495

Jablonka P., Alloin D., Bica E., 1990, A\&A 235, 22

Mayall N., Eggen O., 1953, PASP 65, 24

Mould J., Kristian J., 1986, ApJ 305, 591

Odewahn S., 1987, AJ 92, 311

Ortolani S. Barbuy B., Bica E., 1990, A\&A 236, 362

Pritchet C., van den Bergh S., 1984, PASP 96, 804

Roberts M.S., Whitehurst R.N., 1975, ApJ 201, 327

Sargent W., Kowal C., Hartwick F., van den Bergh S., 1977, AJ 82,947

Sharov A., Lyutyi V., 1983, ApJ 223, 730

Tripicco M., 1989, AJ 97, 735

van Albada S., 1981, ApJ 246, 740

van den Bergh S., 1969, ApJS 19, 145

Vetesnik M., 1962, Bull Astron. Inst. Czech. 13, 180

Zinn R., West M.J., 1984, ApJS 55, 45 